\title{
Development of Injuries Prevention Policies in Mexico: A Big Data Approach
}

\author{
Rosa María Cantón Croda*, Damián Emilio Gibaja Romero \\ Universidad Popular Autónoma del Estado de Puebla (Mexico)
}

Received 15 July 2016 | Accepted 3 February 2017 | Published 27 March 2017

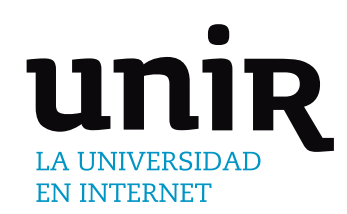

KEYWORDS

Big Data Applications, Decision Theory, Statistical Learning.

$\overline{\text { DOI: } 10.9781 / \text { ijimai.2017.03.005 }}$

\section{INTRODUCTION}

T the past, injuries lacked from government and society attention
because they were considered as an accident and, consequently,
inevitable [1]. Nowadays, health care literature does not longer
contemplate that injuries are inevitable; on the contrary, they can be
prevented when their analysis incorporates intentionality [2]. The
World Health Organization (WHO) considers that an injury is a physical
damage in the human body that results from its exposition to an excess
of mechanical, thermal, chemical or radiant energy. Moreover, this
organization classifies injuries into two types: i) unintentional injuries,
for example, poisoning, road crashes, burns and drowning; and ii)
intentional injuries, which are related to violence from a group or self-
directed [10].

Given that eight of the 15 leading causes of death are injuries, it is important to recall that injuries have an economic impact of societies and governments [31]. The WHO European region report that around $9 \%$ of deaths, and $8 \%$ of hospital admissions, are related to some injury. Within the European Union, the number of deaths by an injury is estimated in 520,000, and a quarter of these are related to intentional injuries [3]. In Latin America, injuries represent the 5\% of their hospital admissions, but, most interestingly, the mortality rate associated with injuries presents an increasing trend among the population between the ages 20 to 40 [34].

Among Latin American countries, the case of Mexico is relevant because its public health system spends around 6 billion dollars in procedures and treatments related to injuries, which represents $1.7 \%$ of Mexican Gross Domestic Product. Specifically, road traffic accidents are the second cause of death in Mexico; and death by some injury is the first cause of death for Mexican population between the ages 30 to 40. It is estimated that 1.1 million of people are involved in some road

* Corresponding author.

E-mail address: rosamaria.canton@upaep.mx traffic in a directed or an undirected way [22].

It is important to recall that injuries do not finish with hospital discharge; most of them generate disabilities that are not well documented because they are not easy to measure. Together with their treatment cost, these disabilities have an adverse impact on individual's work performance, and in his years of healthy life [6, 22]. Together with a high economic cost, physical disabilities may have negative effects on the people's productivity. Although the Mexican government has invested time and resources in different public policies oriented to create a prevention culture, they have failed. The Mexican National Academy of Medicine (MNAM) has identified an increasing trend on hospital admissions attributed to car crashes and unintentional injuries at home, but there is no information about injuries caused by another type of lesion agents $[5,13]$. Even more, recent empirical evidence demonstrates that prevention policies in Mexico have not had the expected effect [24].

In this sense, research literature, on Mexican injuries, focuses on the estimation of costs and trends related to road traffic accidents and gender violence. Key results from these studies point out that those costs have increased since 2000 and prevention policies have not had a significant effect in diminishing the number of injuries associated with road traffic accidents and gender violence [22, 23]. From a geographical aspect, there are descriptive studies that determine focal points where the probability to be bitten or picketed by an animal is high [25].

Recently, [35] analyzes how the public system in Mexico works. They find a lack of efficiency in the treatment of injuries and diseases due to higher costs and limited accessibility, which points out an urgent necessity to change the public health system [27]. However, as far as our knowledge, there are no formal efforts to develop an integral policy oriented to generate a prevention culture to diminish the number of hospital admissions caused by intentional or unintentional injuries.

In this paper, we show all the factors correlated to injuries from the Mexican health system. We analyze the injury database from the Mexican Health Secretary, which includes all relevant information 
such as the type of injury, agent of the lesion, site of occurrence, among others. So, we show the lesion agent that is strongly correlated with the location of occurrence at each federal entity in Mexico. In this sense, our most important contribution relies on offering some insights in the development of specific prevention policies.

The paper is organized as follows. Section 2 shows a brief literature review about how the applications of Big Data to Health. Section 3 describes the methodology used to the paper to collect analyze the injuries database. Section 4 shows our main results, which are the lesion agents strongly correlated to federal entities and sites of occurrence. Also, we discuss some strategies that can be implemented.

\section{LITERATURE REVIEW}

For all, it is well known that Big Data methods are useful for agent's decision-making processes. Although these analytics have been traditionally used for improving the productivity and efficiency within the business, the public sector has incorporated such methods to analyze a huge amount of data that is generated day by day [27, 29]. There is a broad range of public problems where Big Data analytics have been used successfully. For example, during the US president election in 2012, Big Data methods were used to identify the specific needs of voters in the state of Ohio. So, the political campaign of Barack Obama got the victory in Ohio offering a dinner with George Clooney to women between 40 and 49 years old [4].

Consequently, politicians, governments and policy makers are mainly interested in Big Data analytics because they offer the possibility of designing real time strategies against social and economic problems, and natural disasters as well [20,32]. In this sense, our paper is closely related to the literature focused on the improvement of lifestyle. This research pursues the monitoring and analysis of social networks data to establish public policies oriented to improve the public health system and present more detailed health economic studies [14].

\section{A. Big Data and Health}

Given that Big Data methods serve to simplify and identify the most relevant information, researchers and policy makers have pointed out the potential of these techniques in the creation and development of public policies oriented to mitigate or solve specific health problems [11]. Health-care uses Big Data to identify diseases trends and the improvement of life's quality, while preventable deaths are avoided [6]. The model developed by the Harvard University and the Boston Children's Hospital in the USA combine epidemiological information and searches in Google to predict influenza outbreaks one or two weeks earlier than the traditional clinical methods [16]. Although this model over-estimates flu trends, policymakers are interested in the improvement of these practices since their success in the treatment and prevention of Ebola. The use of different types if information contributed with the identification of Ebola hotspots, places where the probability of Ebola occurrence is higher. With these findings, governments allocate more efficiently resources and hospital staff in communities with particular necessities [12]. Pakistan registers another case of success in the application of these techniques in the prevention and correct treatment of dengue. Through a software implementation and the analysis of smartphones interaction, the Pakistani government can identify the residential location of dengue mosquitoes. Consequently, there studies that estimates a saving greater than 200 billion dollars, for the US health system, with the application of these kinds of analytics.

The idea behind the identification of particular diseases trends relies on the fact that people who suffer any discomfort usually search on the Internet what disease is related to their symptoms. With a proper analysis of this information through Big Data methods, health institutions can determine the more accurate treatments for patient's recovery [7]. Even more, the McKinsey Report in 2015 points out that Big Data is transforming the discussion of what is appropriate or inappropriate in diseases treatments that receive a particular population [28]. In other words, with the identification of trends and related factors to specific communities, it is possible to define special treatments based on the characteristics and needs of the community [9]. Consequently, this report identifies the following five contributions of Big Data to health:

- Right living. With a correct analysis of information, it is possible to improve decision making with the promoting of social wellbeing through the engagement of consumers for their care.

- Right care. In other words, it is possible to identify specific treatments and health resources for each patient.

- Right provider. Together with a Right Care, the identification of particular need by communities contributes to the efficient allocation of Care Providers.

- Right value. An effective allocation of resources enhances health care value together with a reduction of costs. Even more, it is possible to do a continuous evaluation of medical institutions while sustainable objectives are pursued.

- Right innovation. Specific community needs require the enhancement of medicine innovation through research and development. Therefore, this new way of information analysis boosts institutions productivity and enhances public social health.

\section{B. Injuries and Public Policy}

Given the economic and social costs associated with injuries treatments, there is an increasing interest in the development of prevention public policies through the identification of the factors that causes an injury [30]. Although the measurement of indirect costs is difficult because a large number of disabilities are not attended, it is possible to identify specific features that contribute to a better definition of prevention policies.

The United States is pioneer and leader in the definition of policies oriented to prevent injuries, both intentional and unintentional. Since the 80 's, American researchers identified the necessity of determining the epidemiology associated with injuries for the design of better public policies. In such decade, death by injuries was the leading cause of mortality in the population of ages from 1 to 40 . For example, there is evidence that injuries associated with soccer decreased in the period from 2004 to 2009, in a comparison between 1990 to 1996 due to the allocation of extra resources in the development and implementation of policies oriented to promote safe practices in soccer [17].

Also, it is well known that the possession of guns for personal security is the primary cause of firearm injuries in the United States (US), where males from minorities are the population more affected. Since the medical and work costs are estimated in 48 billion of dollars, the US government has designed different prevention strategies to diminish the number of injuries related to guns. There is evidence that these programs have contributed in decreasing the number of unintentional firearm injuries in the last twenty years, but intentional gun injuries have increased in recent years [33]. Even more, empirical studies demonstrate that US cities are safer places than rural counties. Consequently, geographical models built on ARGIS program points out the necessity of allocating resources in the countryside. This kind of studies plots twitter and google searches into dynamic maps [28].

For Mexico, the literature of injuries focuses on gender violence and road traffic accidents. There are biological studies that show hot-spots for animal bites in the federal entity of Veracruz [13]. As far as our knowledge, there are no studies that analyze the factors associated with injuries from a Big Data approach. 


\section{MethODOLOGY}

In this work, we analyze data related to injuries following a Big Data approach. Specifically, we follow the method developed by the San Diego Supercomputer Center (SDSC) [7, 31]. This methodology, or data process, is explained in the following subsections.

\section{A. Acquiring Data}

One of the most important aspects of the acquisition of information is to ensure that we have all the data related to our problem. Since injuries have a close relation with health care, we guarantee the previous condition through public databases generated by the Mexican government. Specifically, we get the information from its Ministry of Health, which registers all relevant information related to hospital discharges, injuries, deaths, births, urgencies, population, health resources and health services in the so-called dynamic cubes. So, the injuries cube presents information on injuries such as periodicity and the attention given to patients. This information is classified according to criteria like age, gender, occurrence day, type of lesion, location, among other 55 criteria; and it is available at www.dgis.salud.gob.mx/ contenidos/basesdedatos/bdc_lesiones_gobmx.

It is important to recall that the Mexican Ministry of Health owns information from public and private health institutions in all Mexico, and it is classified following the injury definition established by WHO, i.e. the classification incorporates the intentionality component.

\section{B. Prepare the Data}

During data exploration, we found 62 variables in the dynamic cube of injuries (for a full list, see Appendix 1). Each variable related to injuries can be expanded into 14 options, in average. As we mention before, the Ministry of Health presents information from all the Mexican federal entities, 32 states, and this dynamic cube has information from 2010 to 2014. Although the injuries cube has not been updated in recent years, the Dynamic Cube is the result of a day by day acquisition of information by the Ministry of Health. Consequently, the whole Dynamic Cube has, approximately, a complete data of $14^{62}$ (variables and options) x 32 (states) x 5 (years), which are estimated in $183 \times 10^{71}$.

Since the central aim of this study is to identify the location factors correlated to injuries and their possible trends, in this step we discard 55 from the 62 variables found in the dynamic cube. According to [18], the designing of public health policies must focus on the population welfare. Moreover, public policy should be capable of dealing with specific requirements, i.e., not all regions and individuals require the same treatment. Consequently, geographical location, type of injury and site of occurrence are important variables in the development of a public policy oriented to mitigate this health problem [21].

By the previous discussion, we take the variables year, the federal entity, injury agent, site of occurrence, days, gender and anatomic area of greater severity from the 62 variables registered in the dynamic cube. The values that each of these variables can take are described below:

- Year: 2010, 2011, 2012, 2013, 2014.

- Federal Entity (which establishes the location where an individual receives attention): Aguascalientes, Baja California Sur, Campeche, Coahuila, Colima, Chiapas, Chihuahua, Distrito Federal, Durango, Guanajuato, Guerrero, Hidalgo, Jalisco, México, Michoacán, Morelos, Nayarit, Nuevo León, Oaxaca, Puebla, Querétaro, Quintana Roo, San Luis Potosí, Sinaloa, Sonora, Tabasco, Tamaulipas, Tlaxcala, Veracruz, Yucatán and Zacatecas.

- Injury agent: Fire/flame/hot substance/vapor, drug/drug poisoning, foot/hand, fall, blunt object, sharp object, hit against floor/wall, strange object, explosion, choking/suffocation, multiple agents, projectile gun, hanging, radiation, natural disaster, chemical substances, electric current, tool or machine, shakes, motor vehicle, drowning by submersion, animal picket/bite, forces of nature, poisonous mushroom/plant poisoning, other.

- The site of occurrence: living place, residential institution, school, sports/Athletics area, public road (pedestrian), trade and service area, work, farm, club/canteen/bar, public vehicle, private automotive vehicle, another place, location not specified.

- Day: Monday, Tuesday, Wednesday, Thursday, Friday, Saturday and Sunday.

- Gender: male, female, not specified, unknown.

- Anatomic area of greater severity: Head, face, eye region, neck, spine, superior limbs, hand, chest, back and/or buttocks, abdomen, pelvis, genital region, lower extremities, feet, multiple, others, it is ignored.

Considering previous variables and their values, we work with a database composed by more than 20 million of data, from which nonrelevant information is removed. For example, values like location not specified, gender unknown and anatomic area ignored are not relevant for the designing of public policies. In other words, this kind of values does not offer insights of specific strategies to diminish the occurrence of injuries. This cleaning step contributes to improving the database quality according to the Watson Analytics program.

\section{Explore and Pre-process the Data}

After the cleaning step, we examine the Data Base using the Watson Analytics program. Such tool allows us to summarize and visualize descriptive statistics.

Figure 1 shows the distribution of injuries at each federal entity; it is important to note that Guanajuato is almost the same size than Mexico, which is curious because Mexico is a federal entity four times bigger that Guanajuato, but Guanajuato presents more injuries per capita than Mexico. Consequently, it is necessary to revisit how the public health system works at Guanajuato and Mexico.

Also, Michoacán, Tabasco and Veracruz stand out from Figure 1, which is natural due to the presence of

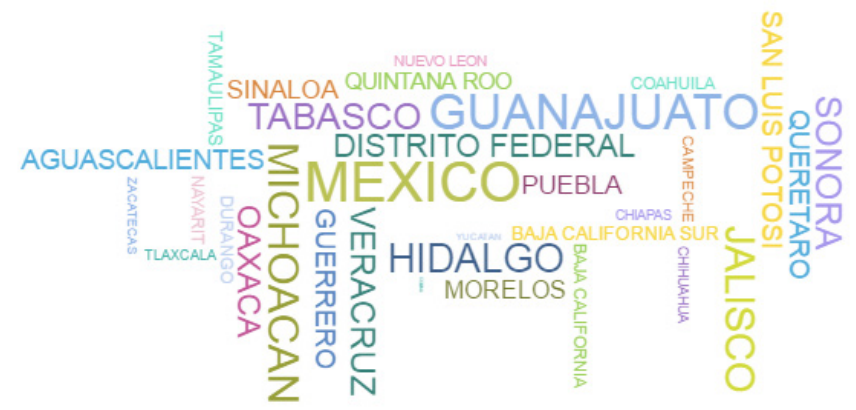

Fig.1. Word-count distribution by Federal Entity, i.e. the size of each federal entity is a function of the number of injury lesions registered as hospital admission at each federal entity.

In a second exploratory exercise, we use Watson Analytics to summarize the distribution of injuries according to the site of occurrence (see Figure 2). We identify that Living Place, Public Road, and Work are the sites where most of the injuries can happen. There is no surprise in the case of Public Road since road traffic accidents are the second cause of death in Mexico. However, Living Place and Work are sites of occurrence where the injuries intentionality matter; from these findings, we can infer a lack of prevention culture prevention in the Mexican population. In contrast, Finland has established specific strategies to generate a prevention culture for injuries at the living place. For example, since older people have a higher probability to suffer a fall in the living place, the National Falls Prevention Program 
in Finland provides with guidelines to prevent falls in these population. Through this program, a person who lives with older people receives instructions to transform a house into a smart home [30]. The United Kingdom and Spain also apply the idea of changing environments into smart environments since ten years ago. Notably, the concept of a smart kitchen has reduced the number of unintentional injuries at the living place in such countries [20]. Also, it is important the European countries and the United States have a long tradition in the prevention of injuries at work. These governments have identified the high correlation between fatigue and work injuries, which has boosted awareness campaigns between workers and employers to determine the risk of injuries by fatigue [26].

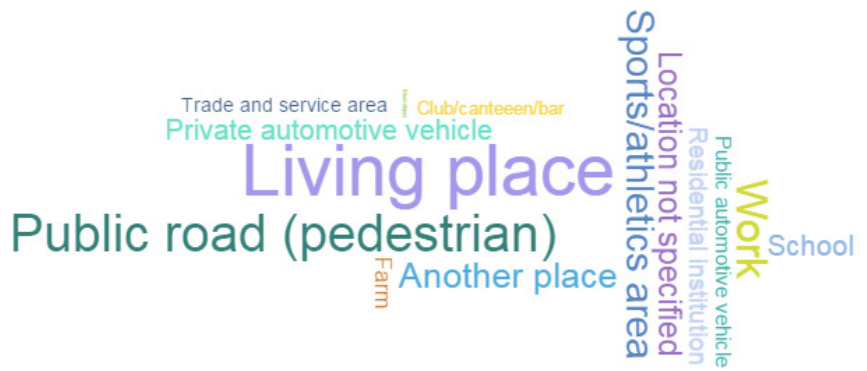

Fig. 2. Word-count distribution by the site of occurrence, i.e. the number of injuries at each location of occurrence determines the size of each possible site of occurrence.

\section{Analyze Data}

The descriptive statistics contribute to the creation of the prediction model in the BigML program. We investigate the correlation between the variables agent of the lesion, federal entity, and site of occurrence. Through the classical experiment of machine learning, we develop an artificial intelligence model. The central aim of this model is the identification of the lesion agent strongly correlated to federal entity and site of occurrence. So, we train our model with the division of our primary database; one with $80 \%$ of the data and the other with the other $20 \%$ of data. Consequently, it was possible to generate a process of knowledge induction through data behavior. So, the BigML analysis follows the following steps:

a) As the Watson Analytics software, we check the database quality, "source quality" with the BigML tools; which report zero missing and zero errors at the "dataset." In other words, the step serves to verify what was done in the cleaning step. Moreover, histograms associated with each variable in the dataset show a regular pattern of the information.

b) The detection of anomalies does not indicate a significant problem in the dataset because they are related to the anatomical area injured, which are not included in the present study. It is important to note that errors associated with anatomical areas are natural since there are instances where injuries case only report a lesion on the head, but not in hands. It is important to recall that anatomical area is not crucial in the definition of prevention policies, but it is an important variable in the allocation of resources and the definition of an efficient mechanism for injuries treatment. In future studies, we will include these variables for a better allocation of health resources.

c) After reviewing the information anomalies, we proceed to generate the regression model through BigML. Thus, the primary data set is divided into two different data sets, one with $80 \%$ of the information and the other with the other $20 \%$. After training the model to verify its reliability, we get the agent lesions that are strongly correlated by the site of occurrence and federal entity.
IV. Results AND Discussions

\section{A. Results}

Our primary results rely on the identification of the Legion agent associated to federal entity and site of occurrence. In other words, the machine learning model identifies the most likely lesion agent by the site of occurrence at each federal entity.

Figure 3 shows in different color the lesion agent correlated with federal entity and site of occurrence; the circle size shows how strong is this correlation. Note that BigML allows the analysis of the whole injuries data set from the Mexican Ministry of Health.

\begin{tabular}{|c|c|c|c|c|c|c|c|c|c|c|c|}
\hline - & 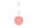 & $\bullet$ & & & & 。 & & & . & & - \\
\hline - & $\bullet$ & 8 & & & & ○ & $\varphi$ & $\bullet$ & - & - & : \\
\hline • & & 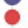 & - & & & & & 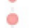 & - & & . \\
\hline 。 & - & 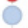 & 。 & & • & 0 & & • & & - & 0 \\
\hline - & & 0 & - & - & $\bullet$ & - & ○ & - & 0 & & $:$ \\
\hline SINALOA & 8 & $:$ & $\bullet$ & & & $\bullet$ & & • & $:$ & 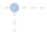 & ? \\
\hline 0 & $\bullet$ & 。 & • & & ○ & $\bullet$ & & $\bullet$ & 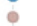 & & . \\
\hline - & - & - & & - & - & - & - & - & - & & • \\
\hline - & - & - & - & & & 0 & - & . & & & 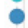 \\
\hline ○ & - & & & $\cdot$ & $\cdot$ & • & ? & • & $\circ$ & & : \\
\hline & & • & & & & - & - & - & & & \\
\hline 0 & & - & - & - & $\circ$ & - & & & & - & $\bullet$ \\
\hline • & & - & & 。 & & 응 & & • & - & . & \\
\hline & $\bullet$ & - & $\bullet$ & & - & ○ & - & • & & • & - \\
\hline ○ & $\bullet$ & 0 & • & - & - & 8 & - & - & - & & e \\
\hline . & : & 8 & - & - & - & 0 & • & $\bullet$ & & - & - \\
\hline 8 & - & - & 0 & - & & 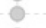 & - & - & & - & - \\
\hline HIDALGO & - & 단 & 은 & - & & 웅 & - & - & - & & - \\
\hline O & - & - & - & - & • & - & - & - & - & & - \\
\hline - & & $\bullet$ & & - & • & & - & - & & & \\
\hline & & - & - & 웅 & - & - & - & $\circ$ & - & & - \\
\hline - & & $:$ & & & & - & - & & & & - \\
\hline • & & - & & & & & - & - & - : & & - \\
\hline 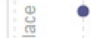 & $\bullet$ & $\bullet$ & 离 & 통 & है & 0 & 点 & 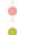 & - & • • & : \\
\hline 竞 & - & - & c & ता & ज्ञ & อల & - 들 & - & 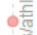 & 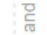 & - \\
\hline • • & • & $\theta$ & ๑ల్ & - & 㥯 & 愛 & $\begin{array}{l}\text { o } \\
\text { g } \\
g\end{array}$ & : & $\bullet$ & 总 & : \\
\hline
\end{tabular}

Fig. 3. Each color represents the agent of lesion strongly correlated with federal entity and site of occurrence.

Although Figure 3 summarizes the lesion agent strongly correlated at the place of occurrence within each federal entity, in this paper, we show the results corresponding to Living Place, Public Road, and Work, the three locations with the higher number of injuries.

The states of Mexico, Jalisco, and Guanajuato are the sites of occurrence with a high probability to be injured at the living place. Also, in these states, there is a high probability that a person will be adversely affected by a hit against wall/floor, chemical substances and animal picket, at her living place. Also, the highest probability to be injured at the Public Road is presented in the states of Michoacán, Tabasco, and Veracruz. It is important to note that the prediction model indicates that Bunt Agent, Projectile Gun and Sharp are the correlated agents of the lesion in those places. Finally, Tabasco, Puebla y Sinaloa are the federal entities where it is most probable to be injured at work. Also, if this happens, the lesion agent associated with these injuries is Hit against Floor/Wall, Animal Picket and Tool/Machine, respectively. Table 1 summarizes previous findings.

\section{B. Discussion}

Although we do not report all the findings in Figure 3, Table 1 shows some interesting facts that can be used in the development of specific prevention policies.

First, note that Mexico is the second state with the higher number of injuries at the living place, and chemical substances are the lesion agent correlated to these lesions; this represents a serious public health problem since Mexico is the most populated federal entity. The literature recognizes the necessity of generating a prevention culture in the management of chemical substances. The University of Tokyo points out that social network is suitable to produce a better management system. Also, from social networks, it is possible to 
TABle I. We Show the First Three Federal Entities that Present a Higher Number of Injuries, and the Lesion Agent Strongly Correlated With THE Site OF OCCURRENCE.

\begin{tabular}{|c|c|c|}
\hline Site of Occurrence & Federal Entity & Most probable lesion agent \\
\hline \multirow{4}{*}{ Living place } & Jalisco & Hit against wall/floor \\
\hline & Mexico & Chemical substances \\
\hline & Guanajuato & Animal Picket \\
\hline & Tabasco & Foot/hand \\
\hline \multirow{4}{*}{ Public road } & Michoacán & Blunt object \\
\hline & Tabasco & Projectile gun \\
\hline & Veracruz & Sharp object \\
\hline & Tabasco & Hit against floor/wall \\
\hline \multirow[t]{2}{*}{ Work } & Puebla & Animal picket \\
\hline & Sinaloa & Tool/Machine \\
\hline
\end{tabular}

disseminate information to improve how chemical substances must be managed at home. Also, these online strategies can serve to diminish the number of injuries attributed to animal picket in Guanajuato. As far as our knowledge, in Guanajuato, the Mexican government implements strategies to prevent dogs bite, but there are no strategies to mitigate scorpions bites [25].

The case of Jalisco is interesting because we find that hit against floor/ wall is the most probable lesion agent at living place. Although there is no convincing explanation for this phenomenon, the literature establishes how smart houses can diminish the number of injuries at home [8]. In other words, prevention policies in Jalisco must be oriented to improve home functionality. Even more, social housing programs have to revisit the design of living places [10].

The results that we get from Public Road injuries are deeply correlated with the violence in Mexico. Michoacán, Veracruz, and Tabasco are among the five states with a higher violence index according to the United Nations International Children's Emergency Fund. Although the Mexican government has implemented strategies action against organized crime since 2006, we find no positive effects on these states. On the contrary, our results suggest that a superior firearm control must be established in Tabasco, while culture and educational strategies have to be implemented in Michoacán and Veracruz, the prediction shows an increasing path to be injured by blunt or sharp object [15].

Finally, it is necessary to revisit workplaces in Tabasco, Puebla, and Sinaloa. The government needs to reconsider if firms at these federal entities satisfy with safety certifications. Also, the literature suggests the encouragement of capacitation programs. This last strategy is strongly recommended to Puebla and Tabasco, who are among the five states with the lower level of education [23].

\section{Conclusion}

By using Watson Analytics to know descriptive data statistics, was possible to visually identify the impact of every one of the variables of analysis in the information of the injuries reported by Health Secretary in Mexico. By using this tools was possible to visually identify the States where injuries happened more; such an as the distribution of the specific places where those injuries occur. BigML simplified the validation of anomalies and the distribution analysis of the data. Also, it was utilized to determine the correlation between Federative entities, the place of occurrence and the agent of the injury. The use of the Chart of Correlations allowed identifying focus issues on each Federative entity.
These results are detailed below, presenting the site of occurrence, the federal entity with more injuries at that site of occurrence and the agent that causes the injury:

Living Place: (Jalisco: Hit against floor/wall), (Mexico: Chemical substances), (Guanajuato: Animal Picket), (Tabasco: Foot/hand), (Tlaxcala: Sharp object), (Veracruz: Choking/Suffocation) (Chiapas: Blunt object).

Public road (Pedestrian): (Michoacan: Blunt object), (Tabasco: Projectile gun), (Veracruz: Sharp object), (Campeche: Fall), (Jalisco: Projectile gun), (Sonora: Strange object). Work (Tabasco: Hit against the floor), (Puebla: Animal picket/bite), (Sinaloa: Tool or Machine), (Michoacan, Blunt object).

This article is a first exploratory study of the information that can be obtained from the Health Secretary and the way that the public policies of the country in question of injury prevention should follow. Analysis of the data shows that the type and agent of the injury go beyond car accidents and gender violence.

\section{APPENDIX}

Variables reported by the Ministry of Health on injuries in Mexico. Primary condition

Agent of injury

Aggressor under the influence of alcohol

Aggressor under illegal drug effects

Aggressor under medical drug effects

Aggressor under no effect

Aggressor under effects is ignored

Single aggressor

Statistical year and month

Anatomic area of greater severity

Safety equipment you used

Motor Vehicle Injury

Used safety equipment

External Cause

Consequence of greater severity

Home

Destination after care

Weekday

Preexisting disability

Aggressor age

Age years patient

Age five years patient

Pregnant patient

Hierarchy

Scholarship

It was holiday

Intentionality

The 100 Municipalities

The 400 Municipalities

Indigenous Peoples

Indigenous reference

Patient under alcohol effects

Patient under illicit drug effects 
Patient under medical drug effects

Patient under no effect

Patient under effects is ignored

Relationship to the affected

Received prehospital care

Responsible for care

Can read and write

The Public Prosecutor's Office was notified

Customer Service

Sex of the aggressor

Sex of the patient

Site of occurrence

Type of care counseling

Type of medical care

Type of care other

Type of psychological care

Type of psychiatric care

Type of surgical care

\section{CLUES}

Name Medical Care Unit

Type of Medical Unit

Medical care unit

User referred by

Violence, neglect and/or neglect

Economic violence

Physical violence

Psychological violence

Sexual violence

Single time violence

\section{ACKNOWLEDGMENT}

We acknowledge the support from the University Popular Autónoma del Estado de Puebla.

\section{REFERENCES}

[1] E. Letouzé, «Big data for development: challenges \& opportunities.,» UN Global Pulse, 2012.

[2] «Los accidentes como problema de salud pública en México,» Intersistemas, 2014.

[3] D. S. \&. F. Racioppi, «The role of public health in injury prevention in the WHO European Region,» International Journal of Injury Control and Safety Promotion, vol. 14, n 4, pp. 271-273, 2008.

[4] M. Joseph, «Big Data and Advanced Analytics for Healthcare,» Prime Dimensions, 2013.

[5] Y. O. Peña, A. S. Carvajal, G. A. Pech, J. H. Santos, R. O. Rodríguez y e. al.., «Risk Factors Associated with Domestic Violence and Homicidal Violence of Women: The Case of Yucatan, Mexico,» Psychology; Irvine, vol. 7, $\mathrm{n}^{\circ}$ 1, pp. 62-73, 2016.

[6] L. A. García, «The efficacy of electronic monitoring in gender violence: criminological analysis,» International E-journal of Criminal Sciences, vol. $1, \mathrm{n}^{\circ} 10,2016$.

[7] C. Crosby, V. Nandigam, M. Phan, C. Youn, C. Baru y R. Arrowsmith, «OpenTopography: Addressing Big Data Challenges Using Cloud Computing, HPC and Data Analytics,» Proceeding od the AGU Fall Meeting, 2014.

[8] P. BSN, R. Linda BSN y H. BSN, «Aging Well With Smart Technology,»
Nursing Administration Quarterly, vol. 29, n 4, pp. 329-338, 2005.

[9] A. Chourasia, M. Wong, D. Mishin, D. R. Nadeau y M. Norman, «A scientific data sharing and collaboration platform,» Proceedings of the XSEDE16 Conference on Diversity, Big Data, and Science at Scale, 2016.

[10] M. D. Keal, N. P. P. Howden-Chapman, C. Cunningham, M. Cunningham, J. Guria, M. G. Bake y e. al, «Home modifications to reduce injuries from falls in the Home Injury Prevention Intervention (HIPI) study: a clusterrandomised controlled trial,» The Lancet, vol. 385, n 9964, pp. 231-238, 2015.

[11] B. Marr, «How Big Data Is Changing Healthcare,» Forbes, 2015.

[12] P. Groves, B. Kayyali y a. S. V. K. D. Knott, «The big-data revolution in health care: Accelerating value and innovation,» Center for US Health System Reform. Business Technology Office. McKinsey \& Company, 201.

[13] R. González, A. Chico, V. Domínguez y G. Iracheta, «Epidemiología de las mordeduras por serpiente. Su simbolismo,» Acta Pediatr Mex, vol. 30, $\mathrm{n}^{\mathrm{o}}$ 3, pp. 182-91, 2009.

[14] Y. Tsuji, K. Tonokura y R. Hayashi, «Chemical substances management systems at the University of Tokyo,» Journal of Environment and Safety, vol. 7, $\mathrm{n}^{\mathrm{o}}$ 2, pp. 129-131, 2016.

[15] S. Bautista y a. M. Magdalena, «Gender and political violence in local governments in the Central Mexican Altiplano,» Politai, vol. 7, $\mathrm{n}^{\circ}$ 12, 2016.

[16] S. Yang, M. Santillana y a. S. C. Kou, «Accurate estimation of influenza epidemics using Google search data via ARGO,» Proceedings of the National Academy of Sciences, vol. 112, no 47, pp. 14473-14478, 2015.

[17] A. Chandran, M. J. Barron, B. J. Westerman y a. L. DiPietro, «Time trends in incidence and severity of injury among collegiate soccer players in the United States,» The American Journal of Sports Medicine, vol. 44, $\mathrm{n}^{\circ}$ 12, 2016.

[18] J. O. Allegrante, R. Mitchell, J. A. Taylor y K. A. Mack, «Injury surveillance: the next generation,» Injury Prevention, $\mathrm{n}^{\circ}$ 22, pp. 63-65, 2016.

[19] A. Pat, K. Larson y a. S. Keshav, «Big-data mechanisms and energy policy design,» Proc. 30th AAAI Conf. Artificial Intelligence, pp. 3887-3893, 2016.

[20] M. Peden, K. McGee y E. Krug, «Injury: A leading cause of the global burden of disease,» Geneva: World Health Organization, 2002.

[21] A. Pentland, T. G. Reid y T. Heibeck, «Big Data: Revolutionizing medicine and public health,» Worl Innovation Summit for Health, 2013.

[22] B. A. Díaz-Apodaca, F. G. D. Cosio, G. Moye-Elizalde y a. F. F. FornelliLaffon, «Egresos por lesiones externas en un hospital de Ciudad Juárez, México,» Comunicación Breve, vol. 31, nº 5, pp. 442-446, 2012.

[23] B. A. Rodríguez y a. M. E. N. González, «Bases para el análisis de la problematización de la inseguridad en México,» Espacios Públicos, vol. 16, no 36, pp. 37-54, 2013.

[24] R. Pérez-Núñez, M. Híjar, A. Celis y a. E. Hidalgo-Solórzano, «El estado de las lesiones causadas por el tránsito en México: evidencias para fortalecer la estrategia mexicana de seguridad vial,» Cadernos de Saúde Pública, vol. 30, $\mathrm{n}^{\circ}$ 5, 2014.

[25] A. L. Cervantes-Trejo, I. Rojas-Vargas y J. S. F.-C. a. Roy, «Trends in traffic fatalities in Mexico: examining progress on the decade of action for road safety 2011-2020,» International Journal of Public Health, vol. 61, $\mathrm{n}^{\mathrm{o}}$ 8, pp. 903-913, 2016.

[26] D. T. Jamison, J. G. Breman y a. A. R. Measham, Disease control priorities in developing countries, Oxford University Press, 2006.

[27] M. D’Agostino, F. Mejía, M. Marti, D. Novillo, F. G. d. Cosío y N. Farach, «Social Dialogue and Scientific Production on Big and Open Data in Health: from Facilitating Behavioral Changes,» International Journal of Health Research, vol. 4, nº 3, pp. 14-22, 2016.

[28] D. W. Bates, S. Saria, L. Ohno-Machado y A. S. a. G. Escobar, «Big Data in Health Care: Using Analytics to Identify and Manage High-Risk and High-Cost Patients,» Health Affairs, vol. 33, nº 7, pp. 1123-1131, 2014.

[29] D. Schooper, J.-D. Lormand y a. R. Waxweiler, «Developing Policies to prevent injuries and violence: guidelines for policy-makers and planners,» World Health, 2006.

[30] National Center for Health Statistics, «Health, United States, 2010: With special feature on death and dying,» Washington, DC, 2011.

[31] P. Kline y a. E. Moreti, «People, Places, and Public Policy: Some Simple Welfare Economics of Local Economic Development Programs,» Annual 
Review of Economics, vol. 6, $\mathrm{n}^{\circ}$ 1, pp. 1-45, 2014.

[32] C. J. L. Murray y et.al., «Disability-adjusted life years (DALYs) for 291 diseases and injuries in 21 regions, 1990-2010: a systematic analysis for the global burden of disease study 2010,» The Lancet, vol. 380, n 9859, pp. 2197-2223, 2014.

[33] M. Peden, K. Oyegbite, J. Ozanne-Smith, A. A. Hyder, C. Branche, A. F. Rahman, F. Rivara y a. K. Bartolomeos, Informe mundial sobre prevención de las lesiones en los niños, UNICEF, 2008.

[34] G.-D. H, C. MV, F.-M. F, B. P y a. et.al., «La carga de la enfermedad en países de América Latina,» Salud Pública México, vol. 53, pp. 72-77, 2011.

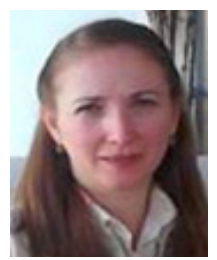

Rosa María Cantón Croda

Rosa María Cantón Croda, Ph.D. in Computer Science from the Tecnológico de Monterrey, Ciudad de México, Master in Information Technologies from the Tecnológico de Monterrey, Veracruz, was graduated in Computer Science Administrator from the Tecnológico de Monterrey, Monterrey. She was Manager of Systems in transport and construction companies. More than twenty years of experience in the academic administration of Tecnológico de Monterrey and currently Dean of postgraduate studies in Engineering and Business at UPAEP. She has written some articles for national and international congresses. She was certified in PMBook, Positive Psychology by Tecmilenio and in Project-Based Learning by Aalborg University in Denmark. Currently, her research interests are Big Data and Business Intelligence.

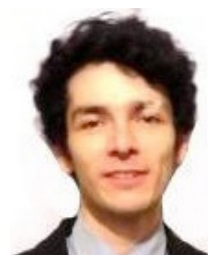

Damián Emilio Gibaja Romero

Damián Emilio Gibaja Romero, Ph.D. in Economics from El Colegio de México, Ciudad de México, Master in Economics from El Colegio de México, México. He has written some articles for national and international congresses. He did research visits at the Paris School of Economics and the University of Glasgow. Currently, hir research interests are Game Theory and Mathematical Economics. 